\title{
APRENDIZAJE BASADO EN PROYECTOS EN FORMACIÓN PROFESIONAL: LA APLICACIÓN DE LAS NUEVAS TECNOLOGÍAS A LA INVESTIGACIÓN DE MERCADOS EN LOS CICLOS DE COMERCIO Y MARKETING
}

\section{PROJECT-BASED LEARNING IN VOCATIONAL TRAINING: THE APPLICATION OF NEW TECHNOLOGIES TO MARKET RESEARCH IN TRADE AND MARKETING STUDIES}

\author{
Fernando González-Ferriz \\ fernando.gonzalez.ferriz@ui1.es \\ Universidad Isabel I. Burgos (España)
}

Recibido: 09/01/2021

Aceptado: 30/06/2021

\begin{abstract}
Resumen:
El presente trabajo de investigación tiene por objetivo profundizar en la metodología del Aprendizaje Basado en Proyectos (ABP) y su aplicación a los estudios de Formación Profesional. Para ello se describen los fundamentos teóricos que dieron origen a la disciplina y se detallan las fases de implementación de un proyecto centrado en la aplicación de las Nuevas Tecnologías a la Investigación de Mercados. Cobran especial atención dos aspectos: el trabajo colaborativo y el proceso de evaluación, aportando ambos las claves de un sistema de enseñanza centrado en el alumno. Esta metodología, si bien requiere cambios, va a contribuir a mejorar los índices de motivación del alumnado, así como el ambiente de trabajo.
\end{abstract}

Palabras Clave: ABP, Aprendizaje Basado en Proyectos, Formación Profesional, Trabajo Colaborativo, Evaluación.

\begin{abstract}
:
The present research pretends to examine the methodology around the Project-Based Learning (PBL) discipline and its application to the vocational training sector. As a result, the paper shows the theoretical foundations in this field of knowledge and carefully details the implementation stages of a project which is focused on the application of New Technologies to Market Research. Special attention is paid to two different aspects: collaborative learning and evaluation processes, which constitute the basis of student-centered education. Although some changes are required, this methodology will contribute to the improvement of two indicators: students' motivation and working conditions in class.
\end{abstract}

Key Words: PBL, Project-Based Learning, Vocational Training, Collaborative learning, Evaluation. 


\section{Introducción}

Si bien el "Aprendizaje Basado en Proyectos" ó "ABP" (del inglés PBL, Project-Based Learning) ha sido analizado por un gran número de académicos en todas las etapas educativas, desde Infantil y Primaria (Molina Torres, 2019; Ramírez et al., 2017; Trujillo, 2015; López y Lacueva, 2008) hasta la Formación Universitaria (Jareño et al., 2020; Toledo y Sánchez, 2018; Ausín et al., 2016; Estruch y Silva, 2006), con especial incidencia en la Educación Secundaria y el Bachillerato (Castro, 2016; Benjumeda et al., 2015; Velez, 2010), también es cierto que no ha acaparado una gran atención en su aplicación a la Formación Profesional, algo que no se entiende muy bien si tenemos en cuenta que uno de los objetivos de este tipo de educación es "el desarrollo de las competencias de innovación y emprendimiento que favorezcan la empleabilidad del alumnado y su desarrollo profesional" (Art. 40 Ley Orgánica 2/2006 de 3 de mayo, LOE, modificado por la Ley Orgánica 8/2013 de 9 de diciembre, LOMCE, y nuevamente por la Ley Orgánica 3/2020 de 29 de diciembre, LOMLOE), aspecto que facilita en gran medida este tipo de formación eminentemente práctica, y basada en el análisis de casos extraídos, en muchas ocasiones, del entorno laboral.

Mucho se ha escrito sobre este tipo de formación, ya que supone un cambio de paradigma en la educación tradicional. Los alumnos dejan de asumir un rol pasivo para convertirse en protagonistas de su propio aprendizaje y "crear" un resultado en base a un problema planteado, aumentando su efectividad y motivación (García et al., 2014; Escribano y Del Valle, 2008; Wurdinger et al., 2007). El profesor, por su parte, también cambia su papel en el aula, pasando a un segundo plano como organizador y coordinador de la actividad. Se pretende, por tanto, que los alumnos sean parte activa del proceso y que interactúen con sus compañeros para resolver problemas de la vida cotidiana, lo cual supondrá una mayor implicación debido a un enfoque mucho más práctico de los módulos formativos. Y si esto es importante en cualquier tipo de educación, todavía lo es más en una Formación Profesional.

Por otra parte, el ABP puede ser un método muy efectivo para la adquisición de las Competencias Clave y las competencias profesionales contempladas en la LOE, LOMCE y LOMLOE, siendo varios los investigadores que apuntan en esta dirección (López, 2020; Escribano y Del Valle, 2008; Martín et al., 2006). Competencias como aprender a aprender, el desarrollo de habilidades sociales, el sentido de iniciativa y espíritu emprendedor, e incluso otras como las competencias digitales, matemáticas o lingüísticas, pueden ser también potenciadas según el tipo de proyecto llevado a cabo.

El objetivo de este trabajo de investigación es profundizar en la aplicación de la metodología del Aprendizaje Basado en Proyectos a los ciclos de Comercio y Marketing, siguiendo para ello la implementación en varias etapas, entre las que destaca el agrupamiento del alumnado, que contribuirá al trabajo colaborativo, y la evaluación de todo el proceso, tanto desde el punto de vista del profesor hacia el alumno, como del alumno hacia este tipo de aprendizaje.

Para ello, este trabajo analizará, en un primer lugar, los fundamentos teóricos que determinan el estado de la cuestión de la temática que se pretende abordar. A continuación, el apartado de metodología desarrollará la puesta en práctica de una propuesta concreta de $A B P$, en base a una serie de etapas, que pretende potenciar la motivación del alumnado en los ciclos de Formación Profesional. Finalmente, las conclusiones mostrarán los resultados más importantes de la ejecución de esa propuesta. 


\section{Fundamentos teóricos}

El ABP parte de una metodología similar basada en problemas, y aunque pueda parecer una metodología relativamente reciente, la realidad es bien distinta, ya que comenzó a utilizarse en los sectores de la arquitectura y la ingeniería en la segunda mitad del siglo XIX. Ya entrado el siglo XX, fueron las universidades norteamericanas las que contribuyeron a su introducción en el mundo académico en la década de los 60, llegando a Europa unos años más tarde con el ánimo de expandirse por todo el continente.

Una de las corrientes académicas sobre las que se asientan, tanto el Aprendizaje Basado en Problemas como en Proyectos, es el constructivismo, que puede ser considerado como una metodología de aprendizaje en la que el individuo representa un papel activo en la construcción de su realidad (Granja, 2015). Por su parte, este enfoque recibirá aportaciones de investigadores de la talla de Piaget, Vygotski y Ausubel, resultando en las teorías constructivistas que conocemos hoy en día (ver Tabla 1). Así pues, el ser humano generará conocimiento nuevo a partir del adquirido anteriormente (Ausubel, 2012; Vygotsky, 1979), y es ahí donde entra en juego este tipo de enseñanza, ya que el alumnado va a ser capaz, gracias a los proyectos desarrollados en clase, de construir nuevo conocimiento, a partir del generado con anterioridad, en ese mismo módulo formativo.

Tabla 1. Aportaciones de Ausubel, Piaget y Vygotsky al constructivismo

\begin{tabular}{|l|l|l|l|}
\hline Autor & \multicolumn{1}{|c|}{ David Ausubel } & \multicolumn{1}{c|}{ JeanPiaget } & \multicolumn{1}{c|}{ Lev Vygotsky } \\
\hline Teoría & $\begin{array}{l}\text { Teoría del aprendizaje } \\
\text { significativo }\end{array}$ & Epistemología genética & $\begin{array}{l}\text { Teoría socio-cultural del } \\
\text { aprendizaje }\end{array}$ \\
\hline Descripción & $\begin{array}{l}\text { Los nuevos conocimientos } \\
\text { se incorporan a la } \\
\text { estructura cognitiva del } \\
\text { individuo ya adquirida. }\end{array}$ & $\begin{array}{l}\text { El conocimiento se } \\
\text { construye partiendo de la } \\
\text { interacción con el medio y } \\
\text { se estructura en distintas } \\
\text { etapas. }\end{array}$ & $\begin{array}{l}\text { El aprendizaje de cada } \\
\text { individuo se basa en el } \\
\text { medio en el que se } \\
\text { interacción con los demás. }\end{array}$ \\
\hline
\end{tabular}

Fuente: elaboración propia

Otra de las contribuciones al fundamento teórico de esta disciplina lo constituye la taxonomía de Bloom (Bloom, 1956), que fue adaptada al nuevo entorno por varios académicos (Wilson, 2006; Anderson y Krathwohl, 2001), con la finalidad de ofrecer un método mucho más planificado, a través de una serie de etapas asociadas a acciones concretas: recordar, comprender, aplicar, analizar, evaluar, y finalmente, crear (ver Fig. 1). Se parte, por tanto, de un pensamiento de orden inferior, para alcanzar uno de orden superior, que consiste en obtener y dar a conocer la solución al problema planteado.

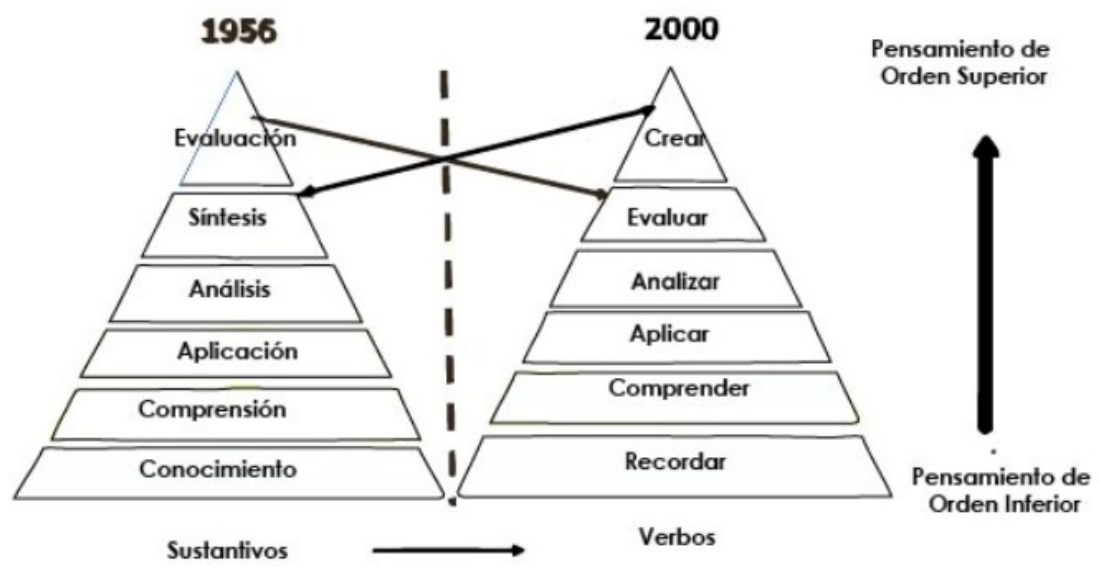

Fig. 1. Adaptación de la taxonomía de Bloom

Fuente: Adaptado de Anderson y Krathwohl, 2001 
Es importante destacar que las teorías constructivistas derivaron en varias corrientes parecidas entre sí pero con algunas diferencias que comentaremos a continuación. El Aprendizaje Basado en Problemas y en Proyectos son las dos tendencias más destacadas, junto con el Aprendizaje Basado en la resolución de Casos. La principal diferencia entre los dos primeros es el grado de libertad y creatividad, ya que, en el aprendizaje por proyectos, los grupos pueden definir un proyecto propio según las especificaciones del docente, resultando en un proceso más creativo. El análisis de casos, por su parte, se centra en la resolución de problemas de la vida real (Bernabeu Tamayo, 2010; Navarro et al., 2008).

En la actualidad, la metodología ABP parte de la base de un método bien estructurado, definido por una serie de etapas, que pueden ser más o menos numerosas en función del enfoque adoptado, entre los que destacan las escuelas de Maastricht o Hong Kong, o el modelo 4x4 (Arpí Miró et al., 2012; Torres, 2020).

Por otra parte, entre las numerosas ventajas que presenta el Aprendizaje Basado en Proyectos para los alumnos, podemos distinguir las siguientes: (1) desarrollo de competencias y destrezas, tales como la colaboración y comunicación, toma de decisiones, o mejor gestión del tiempo, ofreciendo una mejor preparación para afrontar retos futuros, (2) incremento de la motivación al existir una mayor interacción en clase, y como resultado, una mejor predisposición para realizar las tareas asignadas, y (3) fomento del aprendizaje colaborativo, lo cual repercute directamente en un incremento en el nivel de autoestima, contribuyendo a fomentar el respeto y la diversidad en el aula (Vergara, 2016; Jiménez et al., 2013; Sánchez, 2013).

\section{Metodología}

El estudio que aquí se presenta, pretende recoger las experiencias derivadas de la implantación del método de Aprendizaje Basado en Proyectos en los ciclos de Comercio y Marketing en distintos centros de Formación Profesional de la Comunidad Valenciana. Para ello, el proyecto desarrollado consistía en la realización de un estudio de mercado antes del lanzamiento de un determinado producto o servicio, que cada grupo debía definir previamente. Los alumnos disponían de total libertad para elegir el sector de actividad y la información que necesitarían obtener, si bien la obtención y posterior tratamiento de la información se iba a realizar con Google Forms y con herramientas ofimáticas para el tratamiento de la información, que ya eran conocidas por todos ellos (procesador de textos, hoja de cálculo y editor de diapositivas básicamente). Los resultados obtenidos del análisis cuantitativo debían ser procesados y mostrados al resto de la clase, siendo esa presentación objeto de evaluación mediante una rúbrica que había sido entregada previamente a los propios alumnos. En cuanto a los módulos y ciclos donde se implantó esta metodología vienen recogidos en la Tabla 2, junto con la legislación que desarrolla cada uno de los ciclos a nivel nacional y regional.

Tabla 2. Módulos y ciclos formativos a los que se aplica el ABP

\begin{tabular}{|l|l|l|}
\hline Módulo & Ciclo & Legislación \\
\hline $\begin{array}{l}\text { Sistema de } \\
\text { información de } \\
\text { mercados (SIM) }\end{array}$ & $\begin{array}{l}\text { SD 1574/2011, de 4 de noviembre por el que se establece } \\
\text { el título. }\end{array}$ \\
& Internacional (GSCI) & $\begin{array}{l}\text { Orden ECD/319/2012 de } 15 \text { de febrero por la que se } \\
\text { establece el currículo nacional. } \\
\text { Orden 36/2015, de } 30 \text { de marzo, por el que se establece el } \\
\text { currículo en la Comunidad Valenciana. }\end{array}$ \\
\hline
\end{tabular}




\begin{tabular}{|l|l|l|}
\hline Módulo & Ciclo & Legislación \\
\hline & $\begin{array}{l}\text { Superior de Gestión } \\
\text { de Ventas y Espacios } \\
\text { Comerciales (GSGV) }\end{array}$ & $\begin{array}{l}\text { RD 1573/2011, de 4 de noviembre, por el que se establece } \\
\text { el título. } \\
\text { Orden ECD/320/2012, de } 15 \text { de febrero, por la que se } \\
\text { establece el currículo nacional. } \\
\text { Decreto 154/2017, de 6 de octubre, por el que se } \\
\text { establece el currículo en la Comunidad Valenciana. }\end{array}$ \\
\cline { 2 - 4 } $\begin{array}{l}\text { Investigación } \\
\text { Comercial (IC) }\end{array}$ & $\begin{array}{l}\text { RD 1571/2011, de 4 de noviembre, por el que se establece } \\
\text { el título. } \\
\text { Orden ECD/329/2012, de } 15 \text { de febrero, por la que se } \\
\text { establece el currículo nacional. } \\
\text { Superior de } \\
\text { Marketing y } \\
\text { Publicidad (GSMP) } \\
\text { establece el currículo en la Comunidad Valenciana. }\end{array}$ \\
\hline
\end{tabular}

Fuente: elaboración propia

Es importante destacar, que, al igual que ocurre con el resto de actividades que se realizan a lo largo del curso, la "Programación Didáctica" debería recoger con el mayor nivel de concreción posible, los Objetivos y Resultados de Aprendizaje (RA) que se pretenden alcanzar con el ABP, y ahí juega un papel fundamental la legislación, ya que cada uno de los RD que regulan el título correspondiente, recoge tanto las competencias profesionales, personales y sociales, como los Objetivos que se pretenden alcanzar con el título, además de los Resultados de Aprendizaje (RA) y los Criterios de Evaluación (CE) asociados a cada uno de los módulos.

En nuestro caso, si tomamos como ejemplo el módulo de Sistema de Información de mercados (SIM) del ciclo superior de Comercio Internacional, el proyecto, debido a su transversalidad, nos va a permitir consolidar los conocimientos adquiridos a lo largo de todas las Unidades de Trabajo, por lo que se alcanzarán los cinco Resultados de Aprendizaje recogidos en el título, además de los Objetivos relacionados con el módulo de SIM, los Objetivos generales b), ñ), o), p), q), r), s), t), u), v) y w) del ciclo formativo, y las competencias b), m), n), ñ), o), p), q), r) y s) del título (ver Anexo I).

El proyecto se llevó a cabo en una serie de etapas que se detallan a continuación:

\section{Definición del problema}

Esta fase es fundamental para el desarrollo del proyecto porque debe definir de forma clara y concisa el problema planteado. El mayor o menor nivel de concreción supondrá también un mayor o menor grado de libertad a la hora de resolver la actividad y determinará el número de fases que serán comunes a todos los grupos. Así pues, un proyecto sencillo puede plantearse en forma de pregunta abierta, mientras que otro más elaborado deberá presentarse de forma mucho más estructurada. En función del tamaño y la duración del proyecto, también convendrá desglosar el mismo en una serie de tareas que, a su vez, pueden estar compuestas por un determinado número de ejercicios. Por último, es importante destacar que, según la naturaleza del proyecto, se contribuirá al desarrollo de unas u otras capacidades. En nuestro caso, la definición del problema y la descomposición del mismo en tareas y actividades, se muestra de forma esquemática en la tabla 3.

Tabla 3. Definición del problema para una investigación comercial con Google Forms

PROYECTO: INVESTIGACIÓN DE MERCADOS CON GOOGLE FORMS

\begin{tabular}{|c|c|c|}
\hline Pregunt & Curso:2 GS Cl & Módulo: SIM \\
\hline
\end{tabular}
investigación de mercados?

Justificación: A lo largo de su vida profesional, el alumno va a necesitar obtener información del mercado o de los consumidores. Con esta actividad aprenderá cómo hacerlo de forma rápida y efectiva.

RA, CE y CC: $\quad$ RA y CE según RD 1574/2011 de 4 nov.CC: 1-6 


\begin{tabular}{|l|l|}
\hline \multicolumn{2}{|c|}{ TAREA 1: CREACIÓN DE FORMULARIO EN GOOGLE FORMS } \\
\hline ACTIVIDADES & DESCRIPCIÓN \\
\hline 1.Creación formulario & $\begin{array}{l}\text { Redactar el cuestionario con las preguntas correspondientes (abiertas, } \\
\text { cerradas, Likert ...) y trasladarlo a Google Forms. }\end{array}$ \\
\hline 2.Envío formulario & $\begin{array}{l}\text { Compartir el formulario con otros compañeros, profesores y potenciales } \\
\text { consumidores para obtener respuestas. }\end{array}$ \\
\hline 3.Tratam.información & $\begin{array}{l}\text { Una vez obtenidas las respuestas, tratamiento de la información con el propio } \\
\text { formulario de Google o volcando los datos a una hoja de cálculo. }\end{array}$ \\
\hline \multicolumn{1}{|c|}{ TAREA 2: PRESENTACIÓN DE LA INFORMACIóN } \\
\hline \multicolumn{1}{|c|}{ ACTIVIDADES } & $\begin{array}{l}\text { Realizar un informe donde se explique las características de la investigación y } \\
\text { los resultados. Para ello utilizar infografías y procesador de texto. }\end{array}$ \\
\hline $\begin{array}{l}\text { 1.Informe } \\
\text { 2.Presentación }\end{array}$ & $\begin{array}{l}\text { Trasladar las conclusiones más importantes del estudio realizado a una } \\
\text { presentación de diapositivas (Powerpoint, Prezi, Canva...). } \\
\text { Tiempo máx: 10 min. }\end{array}$ \\
\hline \multicolumn{1}{|c|}{ CC adquiridas: 1, 4, 5, 6 } \\
\hline $\begin{array}{l}\text { Leyenda: } \\
\text { RA: Resultados de Aprendizaje, CE: Criterios de Evaluación, CC: Competencias Clave } \\
\text { 1: Aprender a aprender, 2: iniciativa emprendedora, 3: CC. matemáticas, 4: CC. digitales, 5: CC. } \\
\text { sociales, 6: CC. lingüísticas } \\
\text { Nota: Al tratarse de un proyecto transversal se alcanzarán todos los RA (1 a 5) del RD 1574/2011 de 4 } \\
\text { nov., y para su evaluación se trasladarán los correspondientes CE a las rúbricas que se detallan más } \\
\text { adelante. }\end{array}$ \\
\hline
\end{tabular}

Fuente: elaboración propia

II. Agrupamientos y trabajo colaborativo

El trabajo colaborativo es una poderosa metodología que va a contribuir a potenciar la motivación del alumnado, a fomentar varias Competencias Clave (CC), y a mejorar su futura incorporación al mundo laboral. Podríamos recurrir a muchas definiciones, siendo quizás una de las más acertadas aquella que lo describe como el "conjunto de principios y técnicas para ayudar a los alumnos a trabajar juntos con mayor eficacia" (Jacobs et al., 2002).

Las agrupaciones pueden contribuir de forma muy positiva al aprendizaje si se realizan de forma adecuada (Jareño et al., 2014). Para ello deberíamos seguir una serie de criterios basándonos en las aptitudes de cada uno de los alumnos/as. En el caso que nos ocupa, para realizar una investigación comercial utilizando Google Forms, las agrupaciones se podrían llevar a cabo teniendo en cuenta los siguientes factores:

- Número de alumnos por grupo (de forma que no sean demasiados, pero al mismo tiempo los suficientes para poder realizar todas las tareas de forma efectiva). De 3 a 5 por grupo suele ser lo ideal.

- El grado de motivación del alumnado: alumnos más motivados contribuyen de forma muy positiva al funcionamiento del grupo (más incluso que aquellos con mejor rendimiento académico).

- El rendimiento del alumnado: deberíamos mezclar a alumnos/as con distintos comportamientos, habilidades o destrezas y rendimiento académico.

- La multiculturalidad y la igualdad de género: no conviene, por ejemplo, crear equipos sólo de chicos o sólo de chicas, o de una única nacionalidad en el caso de que estuvieran representadas distintas procedencias en el grupo. En su lugar, deberíamos crear grupos que sean lo más heterogéneos posible. 
Las agrupaciones, por tanto, son una poderosa herramienta para cohesionar el grupo, forzando a algunos alumnos/as a salir de su zona de confort y de su grupo habitual de compañeros para trabajar en equipo y desarrollar otro tipo de habilidades. Van a aprender de sus semejantes, y eso resultará muy importante a la hora de considerar otros puntos de vista.

III. Planificación

La planificación en todos los aspectos de la enseñanza y en todas las etapas es una constante en las investigaciones del sector educativo (Burgos y Godino, 2020; Llorent, 2019; Núñez, 2018). En todo proyecto será necesario un proceso de planificación antes de comenzar a trabajar en éste. Es importante saber dónde estamos y dónde queremos llegar, además de realizar una correcta temporalización de las tareas y actividades que componen el mismo. En este contexto, una correcta definición del proyecto para que el alumnado conozca con exactitud qué es lo que se le pide, será fundamental para el buen desarrollo del ABP. Del mismo modo, facilitar a los grupos las rúbricas con los Criterios de Evaluación les va a permitir fijar con más facilidad sus Objetivos, que resultarán clave en el proceso de planificación. Esto es todavía más importante en el caso del Aprendizaje Basado en Proyectos ya que cada grupo de trabajo va a definir su propio escenario, dentro de los límites marcados por el profesor, y será necesario determinar, tanto los resultados que se pretenden alcanzar, como los medios más adecuados para ello.

IV. Investigación

Esta es una de las etapas más creativas del ABP. Una vez definidos los objetivos, los grupos de trabajo deberán utilizar las herramientas necesarias para resolver cada actividad. Si bien en nuestro caso, las herramientas son conocidas por los alumnos (básicamente Google Forms para la creación del cuestionario y una hoja de cálculo para el análisis estadístico de los datos), en otros proyectos podrían disponer de total libertad para la elección de la/s herramienta/s más adecuada/s. Es el momento de desarrollar especialmente las competencias matemáticas y digitales.

\section{Análisis y síntesis}

Una vez llevada a cabo la investigación, los grupos deberán estructurar la información obtenida. Esta es una etapa en la que se potencia de forma especial el trabajo colaborativo, ya que se debe alcanzar un consenso en cuanto a qué aspectos se deberían considerar de cara a la elaboración de la solución final. Se desarrollan, por tanto, de una forma muy especial, las competencias sociales.

\section{Elaboración y presentación de la solución}

Es el momento de "crear" la solución final. Para ello, los distintos grupos han debido seguir el proceso de planificación definido con anterioridad y aplicar los conocimientos teóricos adquiridos a lo largo del curso para alcanzar el objetivo final. Nuevamente la solución debe ser consensuada entre todos los miembros y presentada de forma coherente. En marketing no sólo es importante lo que se dice, sino cómo se dice. En esta ocasión se trabajarán, sobre todo, las competencias digitales, sociales y lingüísticas. En el caso que analizamos, cada grupo expondrá al resto de la clase los resultados obtenidos, ya que los proyectos serán distintos en función del producto o servicio que se haya decidido lanzar al mercado, pero podría obtenerse un resultado agregado que integre el trabajo de todos los grupos siempre que se haya definido el mismo marco de actuación para todos ellos.

Merece la pena dedicar un espacio al uso de herramientas que puedan ser útiles en el Aprendizaje Basado en Proyectos, especialmente en un entorno tan cambiante como el actual. No en vano, son numerosas las investigaciones que profundizan en estos aspectos, prestando cada vez más importancia a nuevas tendencias como la gamificación (Belmonte, 2020; Acosta, 
2018; de la Hoz, 2018; Herán Gascón y Fortunato, 2017; Navarro, 2017). Los organizadores gráficos, por ejemplo, son herramientas visuales no lingüísticas que permiten al alumnado: (1) relacionar nuevos conocimientos con otros adquiridos previamente, (2) descubrir cómo los conceptos se relacionan entre sí y se integran en un contexto general, y (3) recordar la información fácilmente. Destacan por su gran utilidad los mapas visuales y todas las herramientas orientadas a su desarrollo (Mindmeister, Xmind, Mindmanager, Miro...).

Otro tipo de aplicaciones que pueden ser de gran utilidad a la hora de organizar la información gestionada en cada uno de los proyectos son los gestores de información que nos hacen la vida más fácil a la hora de organizar y compartir ficheros: fotos (Google fotos, Instagram, redes sociales), notas (Evernote, Onenote, Google Keep), o cualquier tipo de información (Google Drive, Dropbox, Onedrive). Y por supuesto, aquellos programas y aplicaciones para la elaboración y presentación de las conclusiones obtenidas: presentaciones (Powerpoint, Prezi, Impress, Canva), textos (Word, Writer, Google Docs), vídeos (Openshot, Imovie, Windows MovieMaker), infografías... Todo un mundo de posibilidades a nuestro alcance para hacer del Aprendizaje Basado en Proyectos una experiencia única y altamente enriquecedora.

\section{Evaluación}

A nivel normativo, la evaluación viene regulada en todas y cada una de las leyes educativas. Así pues, la Ley Orgánica 3/2020, de 29 de diciembre (LOMLOE), que acaba de entrar en vigor y modifica la Ley Orgánica 2/2006, de 3 de mayo (LOE), establece que "la evaluación es el proceso que nos va a permitir conocer si los alumnos están adquiriendo los objetivos que nos hemos marcado". Gracias a la evaluación vamos a ser capaces de medir y valorar, tanto el rendimiento de los alumnos, como el del proceso educativo.

A nivel académico, la evaluación es, junto a la metodología, uno de los temas que más interés suscitan. Dos de los aspectos que acaparan un mayor número de investigaciones son la evaluación por competencias (López et al., 2018; Muñoz y Araya, 2017; Exposito-Langa y Tomas Miquel, 2017) y el uso de rúbricas en los procesos de evaluación (Concepción y Medina, 2020; Langa et al., 2017; Velasco-Martínez y Tójar Hurtado, 2017).

En el caso que estamos analizando, la evaluación del alumno se hizo mediante dos rúbricas. La primera (ver tabla 4) se centraba en la tarea 1 (creación de un formulario en Google Forms para el análisis del mercado), y más concretamente en los aspectos relacionados con la resolución del problema en sí. Así pues, las categorías con una mayor ponderación fueron el dominio de los contenidos adquiridos y la aplicación de los mismos a la resolución de los problemas planteados.

Tabla 4. Rúbrica de evaluación de la resolución del problema planteado.

\begin{tabular}{|l|c|l|l|l|l|}
\hline \multicolumn{1}{|c|}{ CATEGORÍA } & POND. & \multicolumn{1}{c|}{ 4(SOBRES.) } & \multicolumn{1}{c|}{ 3(NOTABLE) } & \multicolumn{1}{c|}{ 2(APROBADO) } \\
\hline $\begin{array}{l}\text { CONOCIMIENTO } \\
\text { CONTENIDOS }\end{array}$ & $30 \%$ & $\begin{array}{l}\text { Domina todos los } \\
\text { contenidos del } \\
\text { módulo }\end{array}$ & $\begin{array}{l}\text { Conoce la mayoría de } \\
\text { contenidos del módulo }\end{array}$ & $\begin{array}{l}\text { Solo ha aprendido algunos } \\
\text { contenidos }\end{array}$ & $\begin{array}{l}\text { No domina los } \\
\text { contenidos del módulo }\end{array}$ \\
\hline $\begin{array}{l}\text { APLICACIÓN CONTENIDOS } \\
\text { (definiciones, } \\
\text { procedimientos, cálculos...) }\end{array}$ & $30 \%$ & $\begin{array}{l}\text { Tiene muy buen } \\
\text { dominio de la } \\
\text { aplicación de los } \\
\text { contenidos }\end{array}$ & $\begin{array}{l}\text { Aplica los contenidos de } \\
\text { forma correcta }\end{array}$ & $\begin{array}{l}\text { Aplica los contenidos de } \\
\text { forma básica }\end{array}$ \\
\hline CONCRECIÓN & $20 \%$ & $\begin{array}{l}\text { Se ajusta } \\
\text { perfectamente a lo } \\
\text { pedido en la actividad }\end{array}$ & $\begin{array}{l}\text { Se ajusta a la mayoría de } \\
\text { lo pedido en la actividad } \\
\text { correcta los contenidos }\end{array}$ & $\begin{array}{l}\text { Se ajusta solo en parte a lo } \\
\text { pedido en la actividad }\end{array}$ & $\begin{array}{l}\text { No se ajusta a lo pedido } \\
\text { en la actividad }\end{array}$ \\
\hline $\begin{array}{l}\text { ERPRESIÓN } \\
\text { Y ORTOGRAFÍA }\end{array}$ & $10 \%$ & $\begin{array}{l}\text { Domina la expresión } \\
\text { Se expresa de forma } \\
\text { correcta todo el tiempo }\end{array}$ & $\begin{array}{l}\text { Se expresa de forma } \\
\text { adecuada pero mejorable }\end{array}$ \\
\hline $\begin{array}{l}\text { como la ortografía es } \\
\text { muy buena }\end{array}$ & $\begin{array}{l}\text { La presentación es } \\
\text { buena y la ortografía } \\
\text { bastante buena }\end{array}$ & $\begin{array}{l}\text { La presentación es correcta } \\
\text { y la ortografía aceptable }\end{array}$ & $\begin{array}{l}\text { La presentación es } \\
\text { descuidada y presenta } \\
\text { muchas faltas de } \\
\text { ortografía }\end{array}$ \\
\hline
\end{tabular}

Fuente: elaboración propia 
La segunda rúbrica (ver tabla 5) se centraba en la evaluación de la tarea 2 (presentación de la información), prestando una especial atención al contenido y a la presentación oral de los resultados de la investigación, considerándose aspectos adicionales como la coherencia, la estructura y la originalidad.

Tabla 5. Rúbrica de evaluación de la presentación con el resultado final de la actividad.

\begin{tabular}{|c|c|c|c|c|c|}
\hline CATEGORÍA & POND. & 4(SOBRES.) & 3(NOTABLE) & 2(APROBADO) & 1(INSUFIC.) \\
\hline CONTENIDO & $30 \%$ & $\begin{array}{l}\text { El contenido es el } \\
\text { adecuado y se } \\
\text { ajusta } \\
\text { perfectamente a } \\
\text { la actividad }\end{array}$ & $\begin{array}{l}\text { El contenido es } \\
\text { bueno aunque no } \\
\text { se ajusta en la } \\
\text { totalidad a la } \\
\text { actividad }\end{array}$ & $\begin{array}{l}\text { El contenido es } \\
\text { aceptable aunque } \\
\text { podría ajustarse } \\
\text { mucho más a la } \\
\text { actividad }\end{array}$ & $\begin{array}{l}\text { El contenido no es } \\
\text { adecuado y no se } \\
\text { ajusta a lo pedido } \\
\text { en la actividad }\end{array}$ \\
\hline COHERENCIA & $20 \%$ & $\begin{array}{l}\text { La coherencia de } \\
\text { la presentación es } \\
\text { muy buena }\end{array}$ & $\begin{array}{l}\text { La coherencia de } \\
\text { la presentación es } \\
\text { buena }\end{array}$ & $\begin{array}{l}\text { La presentación } \\
\text { es coherente, } \\
\text { pero puede ser } \\
\text { mejorada }\end{array}$ & $\begin{array}{l}\text { El contenido de la } \\
\text { presentación no } \\
\text { presenta ningún } \\
\text { tipo de } \\
\text { coherencia } \\
\end{array}$ \\
\hline ESTRUCTURA & $10 \%$ & $\begin{array}{l}\text { La estructura es } \\
\text { muy buena } \\
\text { (bloques } \\
\text { relacionados } \\
\text { entre sí) }\end{array}$ & $\begin{array}{l}\text { La estructura es } \\
\text { buena (muestra } \\
\text { diferentes } \\
\text { bloques de forma } \\
\text { correcta) }\end{array}$ & $\begin{array}{l}\text { La estructura es } \\
\text { correcta pero } \\
\text { mejorable }\end{array}$ & $\begin{array}{l}\text { La estructura no } \\
\text { es adecuada y la } \\
\text { presentación } \\
\text { desorganizada }\end{array}$ \\
\hline ORIGINALIDAD & $10 \%$ & $\begin{array}{l}\text { La presentación } \\
\text { es muy original }\end{array}$ & $\begin{array}{l}\text { La presentación } \\
\text { es bastante } \\
\text { original }\end{array}$ & $\begin{array}{l}\text { La originalidad es } \\
\text { aceptable }\end{array}$ & $\begin{array}{l}\text { La presentación } \\
\text { no es original }\end{array}$ \\
\hline $\begin{array}{l}\text { PRESENTACIÓN } \\
\text { ORAL }\end{array}$ & $30 \%$ & $\begin{array}{l}\text { Domina las } \\
\text { técnicas de } \\
\text { presentación y la } \\
\text { comunicación } \\
\text { verbal y no verbal }\end{array}$ & $\begin{array}{l}\text { La presentación } \\
\text { es buena y utiliza } \\
\text { tanto la } \\
\text { comunicación } \\
\text { verbal como no } \\
\text { verbal }\end{array}$ & $\begin{array}{l}\text { La presentación } \\
\text { es correcta pero } \\
\text { muy mejorable }\end{array}$ & $\begin{array}{l}\text { No domina las } \\
\text { técnicas de } \\
\text { presentación }\end{array}$ \\
\hline
\end{tabular}

Fuente: elaboración propia

En ambos casos, las rúbricas presentadas incorporaban los Criterios de Evaluación asociados a los Resultados de Aprendizaje. Como ya se ha comentado, se trata de un proyecto transversal que pretendía alcanzar los cinco Resultados de Aprendizaje incluidos en el RD 1574/2011 de 4 nov., por lo que se tuvieron en cuenta los Criterios de Evaluación asociados a los mismos en aspectos clave como la adecuación de los contenidos, la aplicación de los conocimientos adquiridos a lo largo del curso, o la coherencia del discurso.

Por otra parte, al finalizar el proyecto, se analizó el nivel de consecución de los objetivos planteados con este proyecto a nivel didáctico y la satisfacción de los alumnos. El primero de los aspectos fue evaluado por el profesor, y el segundo por los propios alumnos. Para ello se les suministró un formulario (ver Anexo II) en el que se debían valorar con una escala de Likert entre 0 y 5 aspectos como: (1) el ambiente de trabajo en clase, (2) el método de trabajo utilizado, (3) el proceso de evaluación, (4) la motivación del alumno y (5) su capacidad para trabajar en equipo. Este formulario se entregó en dos momentos distintos del curso: el primero al final de la primera evaluación, y el segundo al finalizar el proyecto que coincidía con el final de la tercera evaluación, y por consiguiente del curso. Los resultados obtenidos, consecuencia de la valoración de estos aspectos por un total de 185 alumnos de distintos cursos, mostraron una mejora media de 0,6 puntos (sobre 5 ) en el ambiente de trabajo en clase y de 0,4 en la motivación del alumnado, aspectos clave del proceso de aprendizaje (ver más detalle en Tabla 6). 
Tabla 6. Resultados del cuestionario de satisfacción y evaluación del proceso de aprendizaje

\begin{tabular}{|l|c|c|c|}
\hline \multicolumn{1}{|c|}{ Ítem } & $\begin{array}{c}\text { Valoración media } \\
\text { primera evaluación }\end{array}$ & $\begin{array}{c}\text { Valoración media } \\
\text { tercera evaluación }\end{array}$ & Diferencia \\
\hline Ambiente de trabajo en clase & 3,9 & 4,5 & $+0,6(12 \%)$ \\
\hline Método de trabajo utilizado & 3,8 & 4,1 & $+0,3(6 \%)$ \\
\hline Proceso de evaluación & 3,8 & 3,9 & $+0,1(2 \%)$ \\
\hline Motivación del alumnado & 4,0 & 4,4 & $+0,4(8 \%)$ \\
\hline Capacidad para trabajar en equipo & 3,7 & 3,9 & $+0,2(4 \%)$ \\
\hline
\end{tabular}

Fuente: elaboración propia

\section{Conclusiones}

Aunque el ABP viene aplicándose a todos los niveles y etapas educativas desde hace varias décadas, es ahora cuando adquiere una importancia especial debido a los altos niveles de motivación e implicación que consigue entre el alumnado (Vergara, 2016; Sánchez, 2013). Ante un cambio de paradigma en el mundo de la educación como el que estamos viviendo en los últimos años, propiciado en gran parte por el rápido avance de las nuevas tecnologías y el inmediato acceso a la información, está claro que es necesario un cambio en la forma de educar a nuestros alumnos. A pesar de ello, todavía encontramos una falta de proyectos de investigación en el entorno de la Formación Profesional, cuestión difícil de entender ya que son precisamente estos estudios los que potencian la adquisición de capacidades y competencias para la incorporación del alumnado al mercado laboral en un futuro cercano.

Uno de los aspectos más importantes del ABP es su base constructivista (Granja, 2015; Ausubel, 2012; Wilson, 2006; Anderson y Krathwohl, 2001), de forma que el individuo adquiere nuevos conocimientos en relación con lo aprendido hasta el momento. Nuevamente, este esquema se ajusta perfectamente al sentido de la Formación Profesional, ya que el alumnado va a tener la posibilidad de desarrollar una serie de proyectos que le permitan relacionar los contenidos teóricos estudiados en clase con la aplicación de los mismos a la resolución de un problema que, en muchas ocasiones, podría encontrarse en el mundo real. Al mismo tiempo, el ABP permitirá alcanzar varias de las Competencias Clave consideradas como prioritarias para alumnos de Secundaria, Bachillerato y Formación Profesional en la nueva Ley Orgánica 3/2020, de 29 de diciembre, más conocida como LOMLOE o "Ley Celaá".

Esta investigación contribuye al análisis del Aprendizaje Basado en Proyectos en la Formación Profesional mediante la estructuración y ejecución de un proyecto para la aplicación de las nuevas tecnologías a la investigación de mercados, siendo éste un aspecto fundamental en los ciclos superiores de Comercio y Marketing. La metodología, que consistente en la implementación de una serie de fases que nos van a permitir "crear" una solución al problema planteado, se centra en una definición lo más precisa posible del mismo, con el fin de determinar unos objetivos concretos que permitan avanzar en el proceso de planificación. Una vez superada la fase de investigación, considerada como la más creativa, que además ofrecerá la posibilidad al alumnado de utilizar herramientas digitales con el fin de combinar teoría y práctica, restará construir la solución y darla a conocer al resto de la clase.

En este proceso, además, no podemos olvidar dos cuestiones que merecen especial atención: el agrupamiento del alumnado y la evaluación de los proyectos y del proceso de aprendizaje. Con respecto a la primera, cabe destacar la importancia del trabajo colaborativo como fuente de motivación y cohesión del grupo (Del Rio et al., 2014; Jacobs et al., 2002), siendo clave para el desarrollo de ciertas Competencias Clave como las sociales y lingüísticas. Y con respecto a la segunda, convendría destacar la importancia del uso de rúbricas ya que van a permitir, además de conocer los Criterios de Evaluación del proyecto, actuar como una herramienta de apoyo en 
el proceso de planificación (Concepción y Medina, 2020; López et al., 2018; Langa et al., 2017). No obstante, la evaluación no sería completa sin la valoración del proceso de aprendizaje por parte de los alumnos. En este sentido, las encuestas arrojan datos muy positivos, con claros incrementos en el nivel de motivación y en la valoración del ambiente de trabajo, al ser comparados dos momentos distintos dentro del mismo curso académico: el final de la primera evaluación, momento en el cual los alumnos ya toman conciencia de la calidad de la formación recibida, y el final de curso, que coincide con la finalización del ABP. Los incrementos en el índice de motivación deben ser considerados de forma muy positiva ya que el perfil del estudiante de ciclos superiores en Formación Profesional suele presentar indicadores ya de por sí elevados al principio de curso al tratarse de una formación voluntaria y no obligatoria. Por otra parte, contribuir a la mejora del ambiente de trabajo debería de ser siempre un objetivo prioritario para cualquier docente.

A pesar de todo lo expuesto, son muchos los autores (Gómez-Pablos et al., 2016; Guitart, 2011; Esteban, 2009) que presentan, de forma conjunta, tanto las ventajas como los inconvenientes de esta metodología de Aprendizaje Basada en Proyectos. Así pues, consideraremos como principales desventajas: (1) las reticencias a introducir cambios en el aula para sustituir una enseñanza tradicional por otra que requiere mayor nivel de adaptación del profesorado, (2) la ausencia de modelos homogéneos y estables, (3) la dificultad para integrar este tipo de educación que requiere de una serie de recursos adicionales en los ya de por sí ajustados contenidos de los módulos formativos, y (4) el diseño de las aulas y centros educativos, que no siempre son propicios para la implementación de estas nuevas metodologías.

A modo de conclusión, es importante destacar que, si bien es cierto que el proceso exige un esfuerzo adicional por parte del docente y una serie de cambios estructurales en los centros, los resultados finales solo muestran aspectos de mejora, sobre todo en especialidades con estudios eminentemente prácticos, como es el caso de la Formación Profesional.

\section{Referencias bibliográficas.}

Acosta, A. H. (2018). Innovación, tecnologías y educación: las narrativas digitales como estrategias didácticas. Killkana sociales: Revista de Investigación Científica, 2(2), 31-38.

Anderson, W. L., David, R., \&Krathwohl, D. R. (2001). Una taxonomía para el aprendizaje, enseñanza y evaluación: una revisión de la taxonomía de Bloom de objetivos educativos. Nueva York: Longman.

Arpí Miró, C., Àvila, P., Baraldés i Capdevila, M., Benito Mundet, H., Gutiérrez del Moral, M. J., OrtsAlís, M., ... \&Rostán Sánchez, C. (2012). El ABP: origen, modelos y técnicas afines. (C) Aula de innovación educativa, 2012, núm. 216, p. 14-18.

Ausubel, D. P. (2012). The acquisition and retention of knowledge: A cognitive view. Springer Science\& Business Media.

Ausín, V., Abella, V., Delgado, V., \&Hortigüela, D. (2016). Aprendizaje basado en proyectos a través de las TIC: una experiencia de innovación docente desde las aulas universitarias. Formación universitaria, 9(3), 31-38.

Belmonte, M. L. (2020). Quien teme perder, ya ha perdido: gamificación en educación superior. In La docencia en la Enseñanza Superior: Nuevas aportaciones desde la investigación e innovación educativas (pp. 32-40). Octaedro.

Benjumeda, F. J., Romero, I., \& López-Martín, M. D. M. (2015). Alfabetización matemática a través del aprendizaje basado en proyectos en secundaria: 163-172. 
Bernabeu Tamayo, M. D. (2010). Estudio sobre innovación educativa en universidades catalanas mediante el aprendizaje basado en problemas y en proyectos. Universitat Autònoma de Barcelona,.

Bloom, B. S. (1956). Taxonomy of educational objectives. Vol. 1: Cognitive domain. New York: McKay, 20, 24.

Burgos, M., \& Godino, J. D. (2020). Modelo ontosemiótico de referencia de la proporcionalidad. Implicaciones para la planificación curricular en primaria y secundaria. Avances de Investigación en Educación Matemática, (18), 1-20.

Castro Martín, P. L. D. (2016). Cartografía autoetnográfica de una genealogía de programas de educación patrimonial desde la perspectiva del aprendizaje basado en proyectos y la investigación-acción.

Concepción, J. F. F., \& Medina, C. R. F. (2020). Una mirada a la evaluación por rúbricas a través de las TIC. Mendive, 18(1), 89-101.

de la Hoz, J. P. (2018). Autoridad docente y Nuevas Tecnologías: cambios, retos y oportunidades. Revista Complutense de Educación, 29(1), 269.

Del Rio, M. C. N., López, C. B., Molina, E. C., \& García, M. G. (2014). Enfoques de atención a la diversidad, estrategias de aprendizaje y motivación en educación secundaria. Perfiles educativos, 36(145), 65-80.

Escribano, A., \& Del Valle, A. (2008). El aprendizaje basado en problemas (ABP). Madrid: Narcea, SA de Ediciones.

Esteban, M. (2009). Un estudio empírico sobre las ventajas e inconvenientes del Aprendizaje Basado en Problemas (ABP) en grupos numerosos. Aprender. Caderno de Filosofia e Psicologia da educaçao, 7, 131-145.

Estruch, V., \& Silva, J. (2006). Aprendizaje basado en proyectos en la carrera de Ingeniería Informática. Actas de las XII Jornadas de la Enseñanza Universitaria de la Informática (JENUI, 2006). Deusto, Bilbao, del, 12, 339-346.

Expósito-Langa, M., Nicolau Juliá, D., \& Tomas Miquel, J. V. (2017). La evaluación por competencias en los grados. Desarrollo de una experiencia en el Grado en Administración y Dirección de Empresas mediante el uso y validación de rúbrica. Revista Complutense de Educación, 28(4), 1155-1171.

García, M. J. G., Otero, J. J. E., \& López, M. C. G. (2014). Experiencia de aplicación de ABP al Grado de Ingeniería Informática. Actas de las XX JENUI. Oviedo, 9(11).

Gómez-Pablos, V. B., del Pozo, M. M., \& Muñoz-Repiso, A. G. V. (2016). Aprendizaje Basado en Proyectos (ABP) incorporando las TIC: ventajas e inconvenientes desde la experiencia del profesorado en ejercicio. In Tecnología, innovación e investigación en los procesos de enseñanza-aprendizaje (pp. 105-113). Octaedro.

Granja, D. O. (2015). El constructivismo como teoría y método de enseñanza. Sophia, (19), 93110.

Guitart, M. E. (2011). Del "Aprendizaje Basado en Problemas"(ABP) al "Aprendizaje Basado en la Acción"(ABA). Claves para su complementariedad e implementación. REDU. Revista de Docencia Universitaria, 9(1), 91.

Herrán Gascón, A. D. L., \& Fortunato, I. (2017). La clave de la educación no está en las nuevas Tecnologías de la Información y la Comunicación (TIC). Acta Scientiarum. Education. 
Jacobs, G. M., Power, M. A., \& Inn, L. W. (2002). The teacher's sourcebook for cooperative learning: Practical techniques, basic principles, and frequently asked questions. Corwin Press.

Jareño, F., Jiménez, J. J., \& Lagos, M. G. (2014). Cooperative learning in higher education: differences in perceptions of contribution to the group. International Journal of Educational Technology in Higher Education, 11(2), 70-80.

Jareño, F., Álamo, R., Lagos, M. G., \& Jiménez, J. J. (2020). Perception of the acquisition of competences for university professors in a context of problem-based learning methodology. International Journal of Management in Education, 14(6), 628-643.

Jiménez, J. J., Lagos, G., \&Jareño, F. (2013). El Aprendizaje Basado en Problemas como instrumento potenciador de las competencias transversales. Revista electrónica sobre la enseñanza de la Economía Pública Págs, 44, 68.

Langa, M. E., Nicolau-Juliá, D., \& Miquel, J. V. T. (2017). La evaluación por competencias en los grados. Desarrollo de una experiencia mediante el uso de rúbrica. Revista complutense de educación, 28(4), 1155-1171.

Llorent, V. J. (2019). Planificación e innovación en educación primaria. Comercial Grupo ANAYA, SA.

López, A. M., \&Lacueva, A. (2008). Projects in a Sixth-Grade Classroom: Entering a bumpy but promising road. EducationalActionResearch, 16(2), 163-185.

López, M. D. C. L., Guerrero, M. J. L., \& García, M. P. P. (2018). El enfoque por competencias en el contexto universitario español. La visión del profesorado. Revista de Investigación Educativa, 36(2), 529-545.

López, D. M. (2020). Experiencia didáctica en la Enseñanza Secundaria. Una propuesta de ABP bilingüe para el desarrollo de las competencias clave. Tendencias pedagógicas, (36), 200-215.

Martín, A. P., Escudero, J. B., Martín, E. R., Sanz, J. M., \& Martín, D. D. (2006). Un nuevo modelo de aprendizaje basado en problemas, el ABP $4 \times 4$ es eficaz para desarrollar competencias profesionales valiosas en asignaturas con más de 100 alumnos. Aula abierta, (87), 171-194.

Molina Torres, M. P. (2019). El Aprendizaje Basado en Proyectos (ABP) en la formación metodológica del profesorado del Grado de Educación Primaria.

Muñoz, D. R., \& Araya, D. H. (2017). Los desafíos de la evaluación por competencias en el ámbito educativo. Educação e Pesquisa, 43(4), 1073-1086.

Navarro, J. A. D., Pérez, E. S. C., \& Marco, M. J. V. (2008). Comparativa entre el aprendizaje basado en proyectos y el aprendizaje basado en problemas. II Jornadas de innovación docente, tecnologıas de la información y de la comunicación e investigación educativa, Zaragoza.

Navarro, G. M. (2017). Tecnologías y nuevas tendencias en educación: aprender jugando. El caso de Kahoot. Opción: Revista de Ciencias Humanas y Sociales, (83), 252-277.

Núñez, H. G. (2018). Modelo de innovación curricular para la planificación sistémica en educación primaria. UCV-HACER: Revista de Investigación y Cultura, 7(3), 11-23.

Ramírez, V., Padial, R., Torres, B., Chinchilla, J. J., Suárez, C., Chinchilla, J. L., ... \& González, M. C. (2017). The effect of a "PBL" physical activity program based methodology on the 
development of values in Spanish Primary Education. Journalof Human Sport and Exercise, 12(4), 1310-1327.

Sánchez, J. (2013). Qué dicen los estudios sobre el Aprendizaje Basado en Proyectos. Actualidad pedagógica.

Toledo Morales, P., \& Sánchez García, J. M. (2018). Aprendizaje basado en proyectos: Una experiencia universitaria. Revista de currículum y formación del profesorado.

Torres, R. O. (2020). Experiencia del Aprendizaje Basado en Proyectos (ABP) en centros universitarios de Ecuador. Revista Estudios en Educación, 3(4), 277-310.

Trujillo, F. (2015). Aprendizaje basado en proyectos. Infantil, Primaria y Secundaria. Ministerio de Educación.

Velasco-Martínez, L. C., \&Tójar Hurtado, J. C. (2017). Uso de rúbricas en educación superior y evaluación de competencias.

Velez, F. (2010). Meeting the needs of English learners in project-based learning schools. University of California, Los Angeles.

Vergara Ramírez, J. J. (2016). Aprendo porque quiero. Editorial SM.

Vygotsky, L. (1979). El desarrollo de los procesos psicológicos superiores. Editorial Crítica, Barcelona. Título en inglés: Mind in society. The development of Higher Psychological processes, 1.

Wilson, L. O. (2006). Beyond bloom-A new version of the cognitive taxonomy. Leslie Owen Wilson's Curriculum Pages.

Wurdinger, S., Haar, J., Hugg, R., \& Bezon, J. (2007). A qualitative study using project-based learning in a mainstream middle school. Improving schools, 10(2), 150-161. 


\section{ANEXO I}

\section{Objetivos, Competencias y Resultados de Aprendizaje (RA) alcanzados con el proyecto} (extraídos de RD 1574/2011, de 4 de Noviembre).

\section{Objetivos generales del ciclo:}

b) Elaborar informes comerciales, aplicando técnicas estadísticas a la información disponible en un SIM, configurando dicho sistema de información de mercados y obteniendo y analizando la información necesaria para la toma de decisiones en la actividad comercial de la empresa en el exterior.

ñ) Analizar y utilizar los recursos y oportunidades de aprendizaje relacionados con la evolución científica, tecnológica y organizativa del sector y las tecnologías de la información y la comunicación, para mantener el espíritu de actualización y adaptarse a nuevas situaciones laborales y personales.

o) Desarrollar la creatividad y el espíritu de innovación para responder a los retos que se presentan en los procesos y en la organización del trabajo y de la vida personal.

p) Tomar decisiones de forma fundamentada, analizando las variables implicadas, integrando saberes de distinto ámbito y aceptando los riesgos y la posibilidad de equivocación en las mismas, para afrontar y resolver distintas situaciones, problemas o contingencias.

q) Desarrollar técnicas de liderazgo, motivación, supervisión y comunicación en contextos de trabajo en grupo, para facilitar la organización y coordinación de equipos de trabajo.

r) Aplicar estrategias y técnicas de comunicación, adaptándose a los contenidos que se van a transmitir, a la finalidad y a las características de los receptores, para asegurar la eficacia en los procesos de comunicación.

s) Evaluar situaciones de prevención de riesgos laborales y de protección ambiental, proponiendo y aplicando medidas de prevención personales y colectivas, de acuerdo con la normativa aplicable en los procesos de trabajo, para garantizar entornos seguros.

t) Identificar y proponer las acciones profesionales necesarias, para dar respuesta a la accesibilidad universal y al "diseño para todos".

u) Identificar y aplicar parámetros de calidad en los trabajos y actividades realizados en el proceso de aprendizaje, para valorar la cultura de la evaluación y de la calidad y ser capaces de supervisar y mejorar procedimientos de gestión de calidad.

v) Utilizar procedimientos relacionados con la cultura emprendedora, empresarial y de iniciativa profesional, para realizar la gestión básica de una pequeña empresa o emprender un trabajo.

w) Reconocer sus derechos y deberes como agente activo en la sociedad, teniendo en cuenta el marco legal que regula las condiciones sociales y laborales, para participar como ciudadano democrático. 


\section{Competencia general del título:}

Planificar y gestionar los procesos de importación/exportación e introducción /expedición de mercancías, aplicando la legislación vigente, en el marco de los objetivos y procedimientos establecidos.

\section{Competencias profesionales, personales y sociales:}

b) Obtener, analizar y organizar información fiable de los mercados internacionales, aplicando las técnicas adecuadas, y establecer un sistema de información eficaz (SIM), que sirva de apoyo en las operaciones de compraventa internacional.

m) Adaptarse a las nuevas situaciones laborales, manteniendo actualizados los conocimientos científicos, técnicos y tecnológicos relativos a su entorno profesional, gestionando su formación y los recursos existentes en el aprendizaje a lo largo de la vida y utilizando las tecnologías de la información y la comunicación.

n) Resolver situaciones, problemas o contingencias con iniciativa y autonomía en el ámbito de su competencia, con creatividad, innovación y espíritu de mejora en el trabajo personal y en el de los miembros del equipo.

ñ) Organizar y coordinar equipos de trabajo con responsabilidad, supervisando el desarrollo del mismo, manteniendo relaciones fluidas y asumiendo el liderazgo, así como aportando soluciones a los conflictos grupales que se presenten.

o) Comunicarse con sus iguales, superiores, clientes y personas bajo su responsabilidad, utilizando vías eficaces de comunicación, transmitiendo la información o conocimientos adecuados y respetando la autonomía y competencia de las personas que intervienen en el ámbito de su trabajo.

p) Generar entornos seguros en el desarrollo de su trabajo y el de su equipo, supervisando y aplicando los procedimientos de prevención de riesgos laborales y ambientales, de acuerdo con lo establecido por la normativa y los objetivos de la empresa.

q) Supervisar y aplicar procedimientos de gestión de calidad, de accesibilidad universal y de "diseño para todos", en las actividades profesionales incluidas en los procesos de producción o prestación de servicios.

r) Realizar la gestión básica para la creación y funcionamiento de una pequeña empresa y tener iniciativa en su actividad profesional con sentido de la responsabilidad social.

s) Ejercer sus derechos y cumplir con las obligaciones derivadas de su actividad profesional, de acuerdo con lo establecido en la legislación vigente, participando activamente en la vida económica, social y cultural.

\section{Resultados de Aprendizaje:}

1. Analiza las variables del mercado y el entorno de la empresa u organización, valorando su incidencia en la actividad de la misma y en la aplicación de las diferentes estrategias comerciales.

2. Configura un sistema de información de marketing (SIM) para la toma de decisiones comerciales, definiendo las fuentes de información, los procedimientos y las técnicas de organización de los datos. 
3. Diseña planes de trabajo para la obtención de información, definiendo los objetivos, las fuentes de información, los métodos y las técnicas aplicables para la obtención de los datos y el presupuesto necesario.

4. Determina las características y el tamaño de la muestra de la población objeto de la investigación, aplicando técnicas de muestreo para la selección de la misma.

5. Realiza el tratamiento y análisis de los datos obtenidos y elabora un informe con los resultados, aplicando técnicas estadísticas y herramientas informáticas.

\section{ANEXO II}

Encuesta de satisfacción y evaluación del proceso de enseñanza.

\begin{tabular}{|l|l|l|l|l|l|}
\hline EVALUACIÓN DEL PROFESOR & 1 & 2 & 3 & 4 & 5 \\
\hline Conocimiento de la materia & & & & & \\
\hline Claridad en la exposición & & & & & \\
\hline Conexión entre teoría y práctica & & & & & \\
\hline Utilidad y calidad de la documentación & & & & \\
\hline Capacidad de comunicación con los alumnos & & & & & \\
\hline Disposición para atender las consultas de los alumnos & & & & & \\
\hline
\end{tabular}

\begin{tabular}{|l|l|l|l|l|l|}
\hline EVALUACIÓN DEL PROCESO DE ENSEÑANZA & 1 & 2 & 3 & 4 & 5 \\
\hline Claridad en los contenidos & & & & & \\
\hline Método de trabajo utilizado en clase & & & & & \\
\hline Adecuación del tiempo & & & & & \\
\hline Adecuación de los ejercicios & & & & & \\
\hline Forma de evaluar & & & & & \\
\hline Satisfacción con el aprendizaje & & & & \\
\hline
\end{tabular}

\begin{tabular}{|l|l|l|l|l|l|}
\hline OTROS ASPECTOS & 1 & 2 & 3 & 4 & 5 \\
\hline Ambiente de trabajo en clase & & & & & \\
\hline Instalaciones y recursos & & & & & \\
\hline Motivación para la asistencia a clase & & & & & \\
\hline Capacidad de trabajo en equipo & & & & & \\
\hline Satisfacción de las expectativas generadas & & & & & \\
\hline Seguimiento y aprovechamiento del curso & & & & \\
\hline
\end{tabular}

